\title{
Double intermittent reward scheduling and secondary reinforcer strength'
}

A discriminated operant procedure was employed to train two groups of rats to activate a nose press in the presence of a tone for food reward, with the latter presented on a continuous or an intermittent schedule. Each group was then divided into two additional groups which were required to learn a lever press response where the only reward was the tone which was presented on a continuous or an intermittent schedule. A durable secondary reinforcer was established which was a function of the intermittent presentation of primary reinforcement during training.

The establishment of a durable and effective secondary reinforcer through the manipulation of reinforcement schedules has recently been an area of controversy, beginning with the work of Zimmerman (1959). But failure to provide control groups and the possible presence of an inadvertent primary reinforcer (Wike, Platt, \& Knowles, 1962) has left Zimmerman's exciting findings in doubt. Fox \& King (1961), adding control groups, found evidence for the superiority of a double intermittent procedure over any procedure involving continuous reinforcement. But a preliminary attempt in our laboratory to replicate the Fox and King study using automated programming equipment resulted in animals which received intermittent primary reinforcement hovering near the food cup, reducing the opportunity for making lever presses.

The present experiment was designed to insure the establishment of a single, measurable functional stimulus as a secondary reinforcer by employing a discriminated operant technique in which animals were required to respond with a nose press upon the presentation of a tone in order to receive food. This technique insured similar mobility of all animals, controlled for stimulus redundancy (Egger \& Miller, 1962), and provided an objective measure of the approach response.

\section{Method}

The Ss were 36 naive, male, albino rats of the Sprague-Dawley strain, approximately 100 days old on the first day of the experiment.

The apparatus consisted of three standard singlelever Gerbrands-Skinner boxes in sound attenuated chambers. Each box was equipped with a standard nose press situated on the back wall of the box. All programming and recording was automatically controlled, and Noyes $.045 \mathrm{gm}$ pellets were used as reinforcers during the training period.

All Ss were reduced to and maintained at an $80 \%$ predeprivation weight. Each animal received 50 food magazine presentations on a VI one minute schedule and $30 \mathrm{~min}$. of nose press training for food. All animals were then given three $1-\mathrm{hr}$. sessions in which the first nose press response in the presence of a tone terminated the tone and provided the reinforcer, but all responses in the absence of the tone produced an additional $30-\mathrm{sec}$. delay in the occurrence of the tone. All animals were then placed on a schedule in which the discriminative stimulus was presented on a VI 30 sec. schedule subject to the restriction that a response in the absence of the tone produced an additional $10-\mathrm{sec}$. delay in the tone presentation. The variable interval presentation was extended to a VI $1 \mathrm{~min}$. schedule subject to the above 10 sec. non-response restriction for a total of 2 more hr. before partial reinforcement training began. The 18 animals receiving partial reinforcers continued to receive 50 tones on a VI $1 \mathrm{~min}$. schedule throughout the remaining training sessions. However, the proportion of nuse press responses made in the presence of the tone resulting in reward was decreased to $60 \%$ for $2 \mathrm{hr}$. of training, $40 \%$ for $2 \mathrm{hr}$., and $10 \%$ for $3 \mathrm{hr}$., with the number of reinforcements given reducing from 50 to 5 . The 18 animals on continuous reinforcement were placed on a VI $2 \mathrm{~min}$. schedule for $2 \mathrm{hr}$., a VI $3 \mathrm{~min}$. schedule for $2 \mathrm{hr}$. and finally, a VI $10 \mathrm{~min}$. schedule for tone presentations for $3 \mathrm{hr}$. This latter procedure had the effect of presenting the tones at the same rate as those actually reinforced in the partial reinforcement group so that equal reinforcements over equal training times was maintained.

For testing the strength of the tone as a secondary reinforcer, a lever was added, and each response

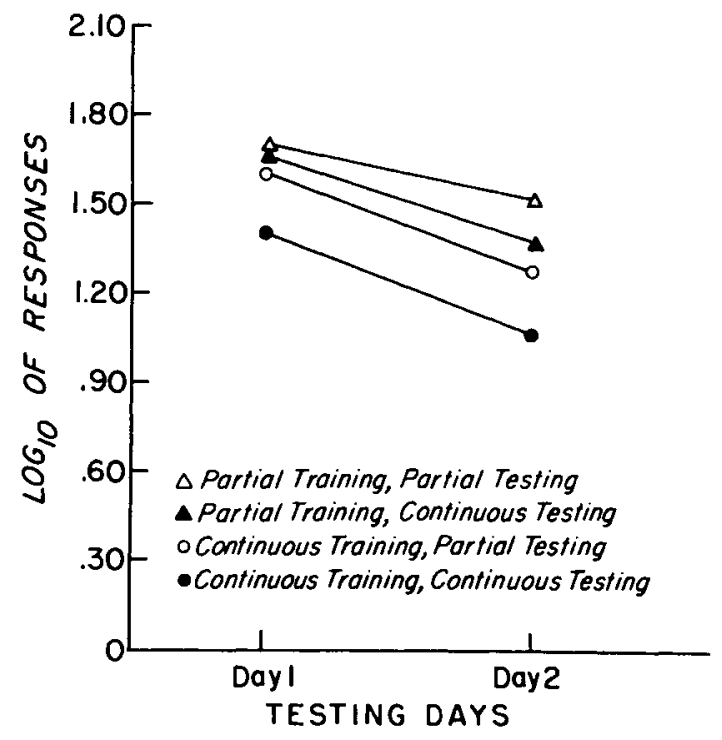

Fig. 1. Mean responses during testing. 


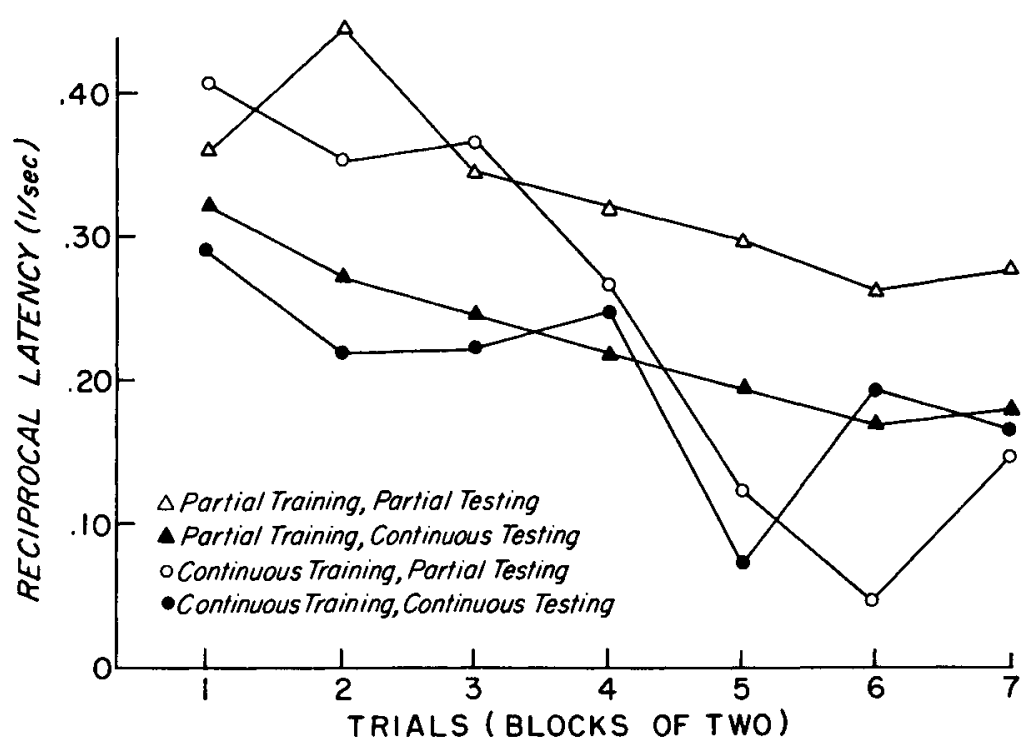

resulted in a tone on a continuous schedule for one half of the group receiving partial pairing during training and a tone on a VI $1 \mathrm{~min}$. schedule for the other half receiving partial pairing during training. A nose press in the presence of the tone terminated the tone. The group receiving continuous pairing of tone and food during training was similarly divided and tested. The number of lever presses and the latency of responding to the tone was recorded for $1 \mathrm{hr}$. on each of two successive days.

\section{Results and Discussion}

An analysis of variance was performed on the reciprocal of the response latencies to the tone on the last day of training, using the median latency to represent an S's performance. There were no significant differences among the four groups $(F=.652 ; p>.25)$, indicating that partial reinforcement during training did not interfere with the continuation of the tone as a discriminative stimulus and that randomization was effective.

The measure of the durability of the tone as a secondary reinforcer was the number of lever responses produced when the only reinforcement was the tone. The response totals for each $\mathrm{S}$ for each of two sessions were transformed to logarithms. Figure 1 shows the mean responses for each group during testing. A between groups, within sessions analysis of the number of responses during testing resulted in significant effects due to training $(\mathrm{F}=4.424 ; \mathrm{p}<.05)$ and to sessions $(F=20.805 ; p<.001)$, indicating a superiority of the group receiving partial primary reinforcement during training, and an overall decrease in responding in the second session. A Duncan's multiple range test indicated that the partial training, partial testing group differed significantly from the continuous training, continuous testing group during the second testing session $(p<.05)$. All other significant differences were between groups during session one and session two.

Figure 2 shows the mean reciprocal latency of responding to the tone during testing for the first 14 tone occurrences (blocks of two). The rank order for the four groups on mean reciprocal latency over the 14 trials was the same as for the secondary reinforcement results. The analysis of variance of this data indicated that the main effect due to tones (testing), trials, and the interaction of training by trials and testing by trials were significant $(F=7.492,2.880,3.294,3.794$; ps $<.01)$. The effects of trials and the interaction of training by trials were as expected, with partial reinforcement training producing a greater durability in fast responding to the tone. The effects involving tones may be due to "massing" of tone production under the continuous reinforcement condition which may have hastened extinction and produced the superiority of the partial tone group where the tone was given on a spaced VI $1 \mathrm{~min}$. schedule.

In conclusion, a durable discriminative stimulus produced a durable secondary reinforcer and the durability was primarily a function of the intermittency of primary reinforcement during discrimination training. Although in the direction expected, the intermittent presentation of the secondary reinforcer during testing produced no significant increase in responding.

\section{References}

Egger, M. D., \& Miller, N. E. Secondary reinforcement in rats as a function of information value and reliability of the stimulus. J. exp. Psychol., 1962, 64, 97-104.

Fox, R. E., \& King, R. A. The effects of reinforcement scheduling on the strength of a secondary reinforcer. J. comp. physiol. Psychol., 1961, 54, 266-269.

Wike, E. L., Platt, J. R., \& Knowles, J. M. The reward value of getting out of a starting box: further extensions of Zimmerman's work. Psychol. Rec., 1962, 12, 397-400.

Zimmerman, D. W. Sustained performance in rats based on secondary reinforcement. J. comp. phy siol. Psychol., 1959, 52, 353-358.

\section{Note}

1. This experiment is based on a thesis submitted by the second author under the guidance of the first author to the Graduate School, Saint Louis University, in partial fulfillment of the requirements for the $M$. $S$. degree. 